\title{
The long road from genotype to phenotype
}

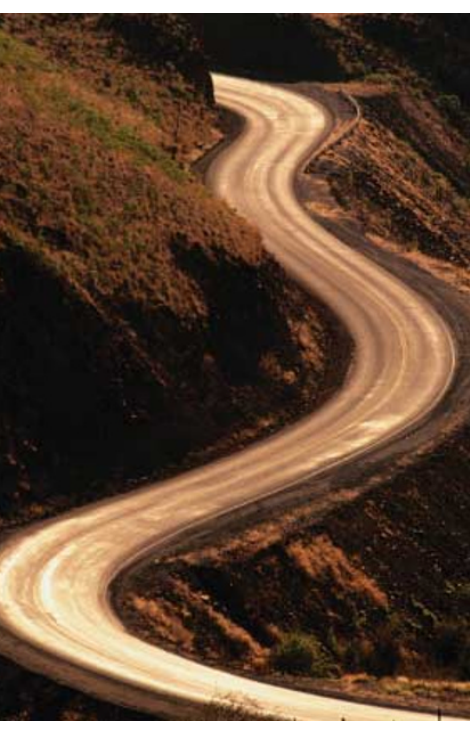

Genetic variation and phenotypic plasticity are key to the evolution of new traits. A recent study by Susan Lindquist and colleagues provides insights into the molecular mechanisms that underlie phenotypic plasticity and shows how the yeast prion $\left[\mathrm{PSI}^{+}\right]$enables cells to tap into their existing genetic variation to acquire complex traits in a single step.

$\left[\mathrm{PSI}^{+}\right]$is formed as a result of a conformational change in the translation termination factor Sup35p. 'Curing' Saccharomyces cerevisiae cells of the prion, which involves growing them in the presence of guanidine hydrochloride and which stabilizes the wild-type Sup35p protein, alters their survival in different growth con- ditions and results in a range of phenotypes, depending on the genetic background. Lindquist and co-workers asked how this genetic diversity is brought about.

They identified three possible explanations. First, curing cells of $\left[\mathrm{PSI}^{+}\right]$also cures them of all other prions, so the phenotypic diversity could result from different strains losing different prions. Second, termination of translation is less efficient in strains that carry $\left[\mathrm{PSI}^{+}\right]$, so readthrough of stop codons might be more frequent in these strains, leading to, for example, translation of pseudogenes or 3' UTRs, which freely acquire genetic variation. Third, protein aggregation, which accompanies
$\left[\mathrm{PSI}^{+}\right]$formation, could affect protein homeostasis.

To test each hypothesis in turn, the authors created yeast strains in which the effects of other prions, translational readthrough and protein aggregation were uncoupled from one another. The results were clear - most of the phenotypic diversity arises as a result of $\left[\mathrm{PSI}^{+}\right]$mediated nonsense suppression.

Outcrossing the $\left[\mathrm{PSI}^{+}\right]$strains to different genetic backgrounds revealed that the newly acquired traits are complex. These heritable complex traits, which can be acquired or lost in as little as a single generation by losing $\left[\mathrm{PSI}^{+}\right]$, can be fixed and maintained even in the absence of the prion. Moreover, a single trait can be fixed in many ways, by reassortment of other genetic polymorphisms or by new mutation, or by a combination of the two.

\section{DEVELOPMENTAL BIOLOGY}

\section{Testing times for Hedgehog}

From the human hand to the fly egg, many anatomical structures acquire their shape thanks to morphogens - form-generating substances that are produced from a localized source and that specify cell identity in a concentration-dependent way. Although morphogens were first proposed 35 years ago, fundamental questions about them persist. Using the Hedgehog $(\mathrm{Hh})$ morphogen as a model, three papers challenge two commonly held assumptions about how morphogens work - coming up with some unexpected conclusions.

The first paper investigated how $\mathrm{Hh}$ concentration is sensed by the cell. The $\mathrm{Hh}$ signalling pathway is unlike most others in that its receptor, Patched $(\mathrm{Ptc})$, is active when not bound to its ligand, as in this state it blocks signalling from the transmembrane protein Smoothened (Smo). Binding of Hh to Ptc relieves this repression and leads to the expression of Smo-target genes. In this deceptively simple interaction, one would assume that ligand-bound Ptc would essentially be equivalent to having no Ptc at all — and that the cell's measure of ambient Hh would be governed simply by the amount of Ptc that remains unliganded. But is this intuitive model true?
Casali and Struhl entertained the alternative hypothesis that the ability of a cell to sense the concentration of $\mathrm{Hh}$ depends on the ratio of bound to unbound Ptc; that is, that the $\mathrm{Hh}-\mathrm{Ptc}$ unit might titrate the inhibitory effect of unbound Ptc on Smo. To distinguish between the two hypotheses, the authors expressed a form of $\mathrm{Ptc}\left(\mathrm{Ptc}^{\mathrm{lloop} 2}\right)$ that cannot bind $\mathrm{Hh}$ and so constitutively represses Smo at three distinct expression levels in the fly wing. They then asked: is the minimum level of $\mathrm{Ptc}^{\Delta l o o p 2}$ necessary to shut down Smo the same, regardless of endogenous Ptc-Hh levels (in which case the traditional model is correct), or does it depend on the presence and amount of the ligand-bound receptor? Their new model was vindicated when they saw that low levels of $\mathrm{Ptc}^{\Delta l o o p 2}$ expression is sufficient to block the pathway in $p t c^{--}$cells, but has little or no effect on normal Hh signalling in otherwise wild-type cells. Instead, high levels of $\mathrm{Ptc}^{\triangle \mathrm{\Delta loop} 2}$ expression was necessary to block Smo activity in the presence of liganded Ptc, indicating some form of titration. Although the model relies on assumptions that remain to be investigated, it seems that Hh-bound Ptc is not the same as having no Ptc at all and that Hh transduction depends on the ratio of bound to unbound Ptc, rather than the absolute amount of unbound Ptc.

Two further papers make an unexpected finding about how the mouse $\mathrm{Hh}$ homologue (sonic hedgehog, Shh) specifies digit identity along the anterior-posterior $(\mathrm{A}-\mathrm{P})$ axis of the forelimb. If Shh were acting by the book, then it would pattern the digits by inducing a linear gradient of Shh responsiveness the effect would be strongest in digit 5 , the most posterior digit that is closest to the morphogen source, and weakest in digit 2 . However, the apparent graded activity of Shh could also arise if cells varied the time they were exposed to a constant level of Shh activity. Two papers report that such a temporal gradient exists and that it acts in conjunction with the canonical spatial gradient. By genetically marking and following the fate of cells that express Shh, Harfe and colleagues show that the temporal gradient arises because cells that are destined to form digits spend a variable time in direct contact with the Shh source before moving anteriorly. Another paper, by Ahn and Joyner, followed the cells that respond to Shh and showed that Shh-responsiveness is not always linear across the A-P axis, but changes over time.

Tempting as it is to say that these results clear up longstanding issues, they are bound to raise further challenges, not least to explain how $\mathrm{Hh} / \mathrm{Shh}$ work in other tissues.

Tanita Casci

\section{(2) References and links} ORIGINAL RESEARCH PAPERS

Casali, A. \& Struhl, G. Reading the Hedgehog morphogen gradient by measuring the ratio of bound to unbound Patched protein. Nature 431, 76-80 (2004) | Ahn, S. \& Joyner, A. L. Dynamic changes in the response of cells to positive Hedgehog signaling during mouse limb patterning. Cell 118, 505-516 (2004) | Harfe, B. D., Scherz, P. J. et al. Evidence for an expansion-based temporal Shh gradient in specifying vertebrate digit identities. Cell 118, 517-528 (2004) 
